\title{
Heat and work fluctuations for a harmonic oscillator
}

\author{
Sanjib Sabhapandit \\ Raman Research Institute, Bangalore 560080, India
}

(Dated: November 10, 2018)

\begin{abstract}
The formalism of Kundu et al. [J. Stat. Mech. (2011) P03007], for computing the large deviations of heat flow in harmonic systems, is applied to the case of single Brownian particle in a harmonic trap and coupled to two heat baths at different temperatures. The large- $\tau$ form of the moment generating function $\left\langle e^{-\lambda Q}\right\rangle \approx g(\lambda) \exp [\tau \mu(\lambda)]$, of the total heat flow $Q$ from one of the baths to the particle in a given time interval $\tau$, is studied and exact explicit expressions are obtained for both $\mu(\lambda)$ and $g(\lambda)$. For a special case of the single particle problem that corresponds to the work done by an external stochastic force on a harmonic oscillator coupled to a thermal bath, the large- $\tau$ form of the moment generating function is analyzed to obtain the exact large deviation function as well as the complete asymptotic forms of the probability density function of the work.
\end{abstract}

PACS numbers: 05.40.-a, 05.70.Ln

\section{INTRODUCTION}

The emergence of the so-called fluctuation relations [1-7] has generated considerable theoretical [8--19] and experimental [20-33] interests in studying fluctuation of various stochastic quantities such as entropy production, heat flow, particle transfer, power injection, work done, etc. in a given time interval $\tau$, in nonequilibrium systems. In this context, one is usually interested in the large- $\tau$ behavior of the probability distribution of the studied stochastic quantity, say $Q$. The probability distribution $P(Q)$ is expected to have a large deviation form [34] $P(Q) \sim \exp [\tau h(Q / \tau)]$ for large $\tau$. However, in spite of the large interests, there are only a few systems where the large deviation function $h(q)$ has been obtained exactly.

An interesting example is a free Brownian particle coupled to two heat baths at different temperatures, initially introduced by Derrida and Brunet [35]. In this model, the quantity of interest is the heat flow from one of the baths to the particle in a given time interval $\tau$. It turns out that this model can be mapped to an exactly solved problem, namely, the quantum harmonic oscillator - Visco [17] has obtained the exact characteristic function $\left\langle e^{-\lambda Q}\right\rangle$ for all $\tau$, and thereafter, the large deviation function $h(q)$ by analyzing the large- $\tau$ limit of $\left\langle e^{-\lambda Q}\right\rangle$.

Now in addition, if a harmonic trap is introduced around the Brownian particle, this seemingly simple model becomes quite non-trivial and any methods for solving the relevant Fokker-Planck equation [Eqs. (7) and (8)] is not known to us. Fortunately, one does not require the complete solution of the Fokker-Planck equation, as the large- $\tau$ behavior is essentially determined by the largest eigenvalue and the corresponding eigenfunctions (left and right) of the Fokker-Planck operator [see Eq. (9)]. It is indeed remarkable that these functions could be found exactly (in terms of some integrals) for a harmonic chain whose two ends are coupled to heat baths at different temperatures [36]. The harmonically bound Brownian particle is a special case of the harmonic chain. In this paper we find the eigenvalue and the eigenfunctions explicitly for the harmonically bound particle, and using those we then find the characteristic function $\left\langle e^{-\lambda Q}\right\rangle$ for large $\tau$. In fact, a special case of this model - that concerns the fluctuations of the work done by an external stochastic force on a harmonic oscillator coupled to a thermal bath - has been realized in a recent experiment [33]. The goal of the this paper is to analyze this problem in detail, as the methods should be useful for other similar problems. Some of the main results have been reported in [37].

This paper is organized as follows. In Sec. II we discuss the problem of the harmonically bound Brownian particle coupled to two heat baths. Section III contains the special case of the harmonic oscillator driven by a random force. In Sec. III A we obtain the large deviation function of the work fluctuation and in Sec. III B we find the complete asymptotic form of the probability density function of the work. Section $[\mathrm{IV}]$ contains some remarks on the evaluation of stochastic integrals that define heat, work, etc., in numerical simulations or from experimental data. Finally we summarize in Sec. V] Some of the details are presented in the appendices. In Appendix A we outline the relevant results of Ref. [36] for the harmonic chain and Appendix A 1 contains some of the details of the bounded Brownian particle case discussed in Sec. III In Appendix B we outline the method of uniform asymptotic expansion of a integral having a saddle-point near a pole, which is used to obtain the complete asymptotic form of the probability density function in Sec. IIIB

\section{BROWNIAN PARTICLE IN A HARMONIC POTENTIAL COUPLED TO TWO THERMOSTATS}

Consider a Brownian particle of mass $m$ in a onedimensional harmonic potential with spring constant $k$ and coupled to two white noise Langevin thermal baths at two different temperatures $T_{L}$ and $T_{R}$ respectively. The displacement $x(t)$ from its mean position and the velocity $v(t)$ of the particle are described by the equations

$$
\dot{x}=v \quad \text { and } \quad m \dot{v}=-\gamma v-k x+\eta(t),
$$

where $\gamma=\gamma_{L}+\gamma_{R}$ and $\eta(t)=\eta_{L}(t)+\eta_{R}(t)$ with $\eta_{L}$ and $\eta_{R}$ being the Gaussian white noises with mean zero and correla- 
tors:

$$
\begin{aligned}
& \left\langle\eta_{L}(s) \eta_{L}(t)\right\rangle=2 d_{L} \delta(s-t) \quad \text { with } d_{L}=\gamma_{L} k_{B} T_{L}, \\
& \left\langle\eta_{R}(s) \eta_{R}(t)\right\rangle=2 d_{R} \delta(s-t) \quad \text { with } d_{R}=\gamma_{R} k_{B} T_{R}, \\
& \text { and }\left\langle\eta_{L}(s) \eta_{R}(t)\right\rangle=0,
\end{aligned}
$$

where $k_{B}$ being the Boltzmann constant. The quantity of interest is the total amount of heat flowing from the reservoir at temperature $T_{L}$ to the particle in a time duration $\tau$ :

$$
Q=\int_{0}^{\tau}\left[\eta_{L}(t)-\gamma_{L} v(t)\right] v(t) d t
$$

This system is a special case $(N=1)$ of the harmonic chain (of $N$ particles) connected at its two ends to reservoirs at different temperatures, that has been studied recently [36] and is outlined in Appendix A.

We consider the restricted characteristic function

$$
Z\left(\lambda, x, v, \tau \mid x_{0}, v_{0}\right)=\left\langle e^{-\lambda Q} \delta[x-x(\tau)] \delta[v-v(\tau)]\right\rangle_{\left(x_{0}, v_{0}\right)}
$$

for fixed initial and final configurations, $\left(x_{0}, v_{0}\right)$ and $(x, v)$ respectively. It satisfies the Fokker-Planck equation [36]

$$
\frac{\partial}{\partial \tau} Z\left(\lambda, x, v, \tau \mid x_{0}, v_{0}\right)=\mathscr{L}_{\lambda} Z\left(\lambda, x, v, \tau \mid x_{0}, v_{0}\right)
$$

with the initial condition $Z\left(\lambda, x, v, 0 \mid x_{0}, v_{0}\right)=\delta\left(x-x_{0}\right) \delta(v-$ $\left.v_{0}\right)$ and the Fokker-Planck operator is given by

$$
\begin{aligned}
\mathscr{L}_{\lambda}=\frac{d_{L}+d_{R}}{m^{2}} \frac{\partial^{2}}{\partial v^{2}}+ & {\left[\frac{k}{m} x+\frac{\gamma+2 \lambda d_{L}}{m} v\right] \frac{\partial}{\partial v} } \\
& -v \frac{\partial}{\partial x}+\lambda\left(\gamma_{L}+\lambda d_{L}\right) v^{2}+\frac{\gamma+\lambda d_{L}}{m} .
\end{aligned}
$$

The solution of the Fokker-Planck equation can be formally expressed in the eigenbases of the operator $\mathscr{L}_{\lambda}$ and the large $\tau$ behavior is dominated by the term having the largest eigenvalue. Thus, for large $\tau$,

$$
Z\left(\lambda, x, v, \tau \mid x_{0}, v_{0}\right) \sim \chi\left(x_{0}, v_{0}, \lambda\right) \Psi(x, v, \lambda) e^{\tau \mu(\lambda)},
$$

where $\Psi(x, v, \lambda)$ is the eigenfunction corresponding to the largest eigenvalue $\mu(\lambda)$ and $\chi\left(x_{0}, v_{0}, \lambda\right)$ is the projection of the initial state onto the eigenstate corresponding to the eigenvalue $\mu(\lambda)$. These functions are obtained in Appendix A 1 and we find that

$$
\begin{gathered}
\mu(\lambda)=\frac{1}{2 \tau_{\gamma}}[1-\eta(\lambda)], \\
\Psi(x, v, \lambda)=\left[\frac{\gamma \eta(\lambda) \sqrt{k m}}{2 \pi\left(d_{L}+d_{R}\right)}\right] \exp \left[-B_{+}(\lambda) E(x, v)\right], \\
\text { and } \chi\left(x_{0}, v_{0}, \lambda\right)=\exp \left[-B_{-}(\lambda) E\left(x_{0}, v_{0}\right)\right],
\end{gathered}
$$

where $\tau_{\gamma}=m / \gamma$ is the viscous relaxation time,

$$
\begin{aligned}
& \eta(\lambda)=\sqrt{1+4 \frac{d_{L} d_{R}}{\gamma^{2}} \lambda(\Delta \beta-\lambda),} \\
& E(x, v)=\frac{1}{2} k x^{2}+\frac{1}{2} m v^{2},
\end{aligned}
$$

and $B_{ \pm}(\lambda)$ is given by Eq. A27. Fogedby and Imparato have recently shown [38] that $\mu(\lambda)$ can also be obtained by the Derrida-Brunet method [35].

Using the explicit forms one can easily verify that $\mathscr{L}_{\lambda} \Psi(x, v, \lambda)=\mu(\lambda) \Psi(x, v, \lambda)$. Moreover, since from (A27) we get $B_{+}(\lambda)+B_{-}(\lambda)=\gamma \eta(\lambda) /\left(d_{L}+d_{R}\right)$, it immediately follows that

$$
\int_{-\infty}^{\infty} \int_{-\infty}^{\infty} \chi(x, v, \lambda) \Psi(x, v, \lambda) d x d v=1,
$$

which is demanded by the normalization. From the above expressions, we also find that $\mu(0)=0$ and $\chi\left(x_{0}, v_{0}, 0\right)=1$. Since $\lambda=0$ case of Eq. (6) gives the probability distribution of the phase-space variables and $\mu(0)$ is the largest eigenvalue, it follows from Eq. (9) that $\Psi(x, v, 0)$ is the steady-state distribution of the phase-space. Therefore, averaging over the initial variables $\left(x_{0}, v_{0}\right)$ with respect to $\Psi\left(x_{0}, v_{0}, 0\right)$ and integrating over the final variables $(x, v)$, we find the characteristic function of the heat flow in the steady state as

$$
Z(\lambda, \tau)=\left\langle e^{-\lambda Q}\right\rangle \approx g(\lambda) e^{\tau \mu(\lambda)},
$$

where

$$
g(\lambda)=\frac{4 \eta(\lambda)}{[1+\eta(\lambda)]^{2}-\left[2 \lambda d_{L} / \gamma\right]^{2}}
$$

Interestingly, both $\mu(\lambda)$ and $g(\lambda)$ are independent of the spring constant $k$. However, while $\mu(\lambda)$ is same for both $k \neq 0$ and $k=0$ cases (the latter was obtained in Ref. [17]), $g(\lambda)$ for $k \neq 0$ differs from that for $k=0$. More precisely, $\left.g(\lambda)\right|_{k \neq 0}=$ $\left[\left.g(\lambda)\right|_{k=0}\right]^{2}$. The $k \rightarrow 0$ limit of $g(\lambda)$ is not same as the $k=$ 0 case. Therefore, although the large deviation function are same for both the cases, the precise asymptotic form of the probability density functions of $Q$ are different.

The leading behavior of the probability density function $P(Q) \sim \exp [\tau h(Q / \tau)]$ can be obtained by inverting Eq. (16) using the saddle-point approximation. Usually the prefactor $g(\lambda)$ can be ignored in such calculation and the large deviation function $h(q)$ is related to $\mu(\lambda)$ by the Legendre transform

$$
h(q)=\mu\left(\lambda^{*}\right)+\lambda^{*} q, \quad-\mu^{\prime}\left(\lambda^{*}\right)=q .
$$

However, if $g(\lambda)$ has any singularities in the region of the saddle-point integration, the functions $h(q)$ and $\mu(\lambda)$ are not simply related by the Legendre transform, and it it important to retain the prefactor $g(\lambda)$ in the saddle-point calculation [8, 9, 17], as we see in the next section where we consider a special case that corresponds to an experiment reported in Ref. [33].

\section{HARMONIC OSCILLATORS DRIVEN BY AN EXTERNAL RANDOM FORCE}

Consider a harmonic oscillator coupled to a thermal bath and driven out of equilibrium by an external Gaussian random 
force. The displacement $x(t)$ of the harmonic oscillator from its mean position is described by the Langevin equation

$$
m \frac{d^{2} x}{d t^{2}}+\gamma \frac{d x}{d t}+k x=\zeta_{T}(t)+f_{0}(t),
$$

where $m$ is the mass, $\gamma$ is the viscous drag coefficient and $k$ is the spring constant. The interaction with the thermal bath is modeled by a Gaussian white noise $\zeta_{T}(t)$ with zero-mean $\left\langle\zeta_{T}(t)\right\rangle=0$. The externally applied force $f_{0}(t)$ is again a Gaussian random variable with $\left\langle f_{0}(t)\right\rangle=0$, and $\zeta_{T}$ and $f_{0}$ are uncorrelated. Equation 19 is asymmetric in $\zeta_{T}$ and $f_{0}-$ the fluctuation-dissipation theorem relates the thermal fluctuation to the viscous drag as $\left\langle\zeta_{T}(s) \zeta_{T}(t)\right\rangle=2 D \delta(s-t)$ where $D=\gamma k_{B} T$ with $T$ being the temperature of the bath and $k_{B}$ being the Boltzmann constant, whereas the fluctuation of the external force $\left\langle f_{0}(s) f_{0}(t)\right\rangle=\left(\boldsymbol{\delta} f_{0}\right)^{2} \boldsymbol{\delta}(s-t)$ is independent of $\gamma$. The quantity of interest is the work done by the external random force $f_{0}(t)$ on the harmonic oscillator in a time interval $\tau$, in the nonequilibrium steady state. This is given (in units of $k_{B} T$ ) by

$$
W_{\tau}=\frac{1}{k_{B} T} \int_{0}^{\tau} f_{0}(t) \frac{d x}{d t} d t
$$

with the initial condition (at $\tau=0$ ) drawn from the steady state distribution.

It is evident that this harmonic oscillator problem can be mapped to the problem of the Brownian particle discussed in the previous section, with the following set of transformations:

- $\eta_{L}(t) \rightarrow f_{0}(t)$ with $\gamma_{L} \rightarrow 0, T_{L} \rightarrow \infty$ while keeping $\gamma_{L} T_{L}=d_{L}$ fixed and $d_{L} \rightarrow\left(\delta f_{0}\right)^{2} / 2$.

- $\eta_{R}(t) \rightarrow \zeta_{T}(t)$ with $T_{R} \rightarrow T, \gamma_{R} \rightarrow \gamma$, and $d_{R} \rightarrow D$.

Under these transformations, we have $Q \rightarrow(\gamma / D)^{-1} W_{\tau}$. Therefore, using Eq. 16] we now get

$$
\left\langle e^{-\lambda W_{\tau}}\right\rangle \approx g(\lambda) e^{\tau \mu(\lambda)},
$$

where $\mu(\lambda)$ and $g(\lambda)$ are given by Eq. 10 and Eq. 17p respectively with the transformation $\lambda \rightarrow(\gamma / D) \lambda$.

It is useful to express the relative strength of the external force with respect to the thermal noise in terms of the dimensionless parameter

$$
\alpha=\frac{\left(\delta f_{0}\right)^{2}}{2 D}=\frac{\left\langle x^{2}\right\rangle}{\left\langle x^{2}\right\rangle_{\mathrm{eq}}}-1, \quad \text { and } \alpha \in(0, \infty),
$$

where $\left\langle x^{2}\right\rangle$ and $\left\langle x^{2}\right\rangle_{\text {eq }}$ are the variance of $x$ in the nonequilibrium steady state (for $f_{0} \neq 0$ ) and in equilibrium (for $f_{0}=0$ ) respectively. Using this parameter $\alpha$ and the above transformations, the expression of $\eta(\lambda)$ given by (13) becomes

$$
\eta(\lambda)=\sqrt{1+4 \alpha \lambda(1-\lambda)} .
$$

We also rewrite $g(\lambda)$ as

$$
g(\lambda)=\frac{2}{1+\eta(\lambda)-2 \alpha \lambda} \times \frac{2 \eta(\lambda)}{1+\eta(\lambda)+2 \alpha \lambda},
$$

where, the first factor in the above equation is due to the averaging over the initial conditions with respect to the the steady state distribution and the second factor is due to the integrating out of the final degrees of freedom.

The probability density function of the work done is related to its characteristic function by the inverse Fourier transform

$$
P\left(W_{\tau}=w \tau\right) \approx \frac{1}{2 \pi i} \int_{-i \infty}^{+i \infty} g(\lambda) e^{\tau f_{w}(\lambda)} d \lambda,
$$

where

$$
f_{w}(\lambda)=\frac{1}{2}[1-\eta(\lambda)]+\lambda w
$$

and we have set $\tau_{\gamma}=1-$ this is equivalent to redefining the time in the unit of $\tau_{\gamma}$, i.e., $\tau / \tau_{\gamma} \rightarrow \tau$. The integration is done along the imaginary axis (vertical contour through the origin) in the complex- $\lambda$ plane.

\section{A. The large deviation function}

The large- $\tau$ behavior of $P\left(W_{\tau}\right)$ can be obtained from the saddle point approximation of the integral in Eq. 25). The saddle-point $\lambda^{*}$ is obtained from the solution of the condition $f_{w}^{\prime}\left(\lambda^{*}\right)=0$ as

$$
\lambda^{*}(w)=\frac{1}{2}\left[1-\frac{w}{\sqrt{w^{2}+\alpha}} \sqrt{1+\frac{1}{\alpha}}\right] .
$$

It follows from the above expression that $\lambda^{*}(w)$ is a monotonically decreasing function of $w$ (see Fig. 11$)$ and $\lambda^{*}(w \rightarrow$ $\mp \infty) \rightarrow \lambda_{ \pm}$, where

$$
\lambda_{ \pm}=\frac{1}{2}\left[1 \pm \sqrt{1+\frac{1}{\alpha}}\right] .
$$

Therefore, $\lambda^{*} \in\left(\lambda_{-}, \lambda_{+}\right)$. The $\alpha$ dependence of $\lambda_{ \pm}$are displayed in Fig. 22 We note that $\eta(\lambda)$ can be written in terms of $\lambda_{ \pm}$as

$$
\eta(\lambda)=\sqrt{4 \alpha\left(\lambda_{+}-\lambda\right)\left(\lambda-\lambda_{-}\right)} .
$$

Clearly, $\eta(\lambda)$ has two branch points on the real- $\lambda$ line at $\lambda_{ \pm}$, and $\eta(\lambda)$ is real and positive for $\lambda \in\left(\lambda_{-}, \lambda_{+}\right)$. Therefore, $f_{w}(\lambda)$ is also real on the real- $\lambda$ line in the interval $\left(\lambda_{-}, \lambda_{+}\right)$. At $\lambda=\lambda^{*}$ we find

$$
\eta\left(\lambda^{*}\right)=\frac{\sqrt{\alpha(1+\alpha)}}{\sqrt{w^{2}+\alpha}} .
$$

Let us now look at the analyticity of $g(\lambda)$ given by Eq. 24) for $\lambda \in\left(\lambda_{-}, \lambda_{+}\right)$. It is clear that $g(\lambda)$ is real for $\lambda \in\left(\lambda_{-}, \lambda_{+}\right)$. From Eq. (28) we find that

$$
2 \alpha \lambda_{-}+1=\sqrt{1+\alpha}[\sqrt{1+\alpha}-\sqrt{\alpha}]>0 .
$$

Therefore, $1+\eta(\lambda)+2 \alpha \lambda>0$ for $\lambda \in\left(\lambda_{-}, \lambda_{+}\right)$for all $\alpha \in$ $(0, \infty)$. Again, from Eq. 28, we find that

$$
2 \alpha \lambda_{+}-1=\sqrt{\alpha(1+\alpha)}-(1-\alpha) .
$$




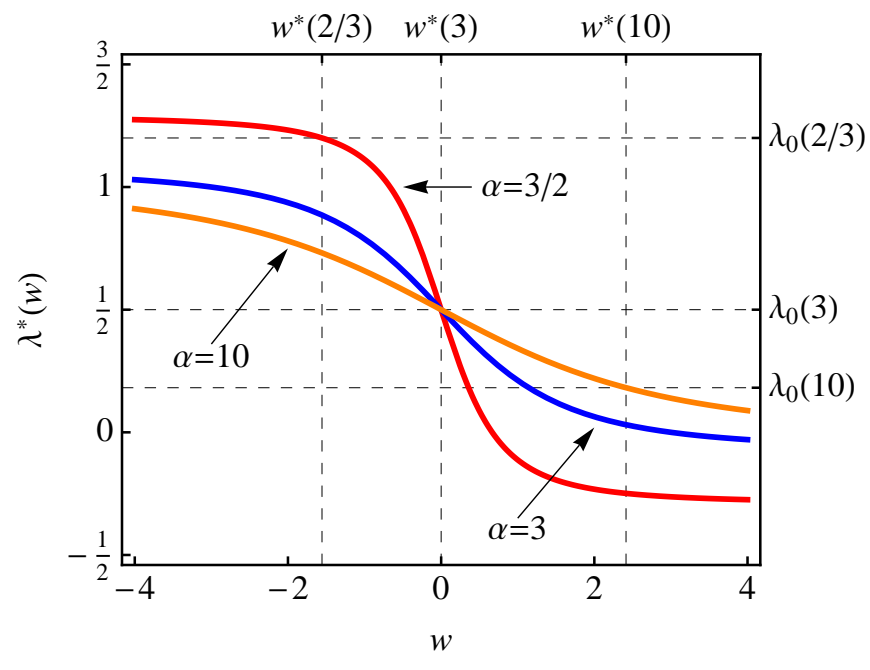

FIG. 1. (Color online). Plots of the saddle-point $\lambda^{*}$ as a function of $w$ for $\alpha=3 / 2$ (red), 3 (blue) and 10 (orange). The horizontal dashed lines mark the positions of the pole $\lambda_{0}$. The vertical dashed lines mark $w^{*}$ for respective values of $\alpha$.

The right side of the above equation is negative for $\alpha<1 / 3$. Thus, $1-2 \alpha \lambda>0$ for $\lambda \leq \lambda_{+}$and $\alpha<1 / 3$. Consequently, when $\alpha<1 / 3$, we have $1+\eta(\lambda)-2 \alpha \lambda>0$ for $\lambda \in\left(\lambda_{-}, \lambda_{+}\right)$. Therefore, $g(\lambda)$ does not have any singularities in the interval $\left(\lambda_{-}, \lambda_{+}\right)$as long as $\alpha<1 / 3$. Ignoring the subleading contribution $g(\lambda)$ in the saddle-point calculation gives

$$
P\left(W_{\tau}=w \tau\right) \sim e^{\tau h_{1}(w)}
$$

with

$$
h_{1}(w)=f_{w}\left(\lambda^{*}\right)
$$

By substituting the expression of $\lambda^{*}$ in $f_{w}\left(\lambda^{*}\right)$, after some algebra, we find that

$$
h_{1}(w)=\frac{1}{2}\left[1+w-\sqrt{w^{2}+\alpha} \sqrt{1+\frac{1}{\alpha}}\right] .
$$

We now consider the case $\alpha \geq 1 / 3$. In this case, it is useful to express $g(\lambda)$ in the form

$$
g(\lambda)=-\frac{\eta(\lambda)[\eta(\lambda)+2 \alpha \lambda-1]}{\alpha(1+\alpha) \lambda[1+\eta(\lambda)+2 \alpha \lambda]} \frac{1}{\lambda-\lambda_{0}},
$$

where

$$
\lambda_{0}=\frac{2}{1+\alpha} .
$$

and $\lambda_{-}<0<\lambda_{0} \leq \lambda_{+}$(see Fig. 2). In order for $\lambda_{0}$ to be a zero of $1+\eta(\lambda)-2 \alpha \lambda$, it has to satisfy the condition $2 \alpha \lambda_{0}-1=$ $\eta\left(\lambda_{0}\right) \geq 0$. Using the above expression of $\lambda_{0}$ we get

$$
\eta\left(\lambda_{0}\right)=2 \alpha \lambda_{0}-1=\frac{3 \alpha-1}{1+\alpha} .
$$

Therefore, $g(\lambda)$ possesses a simple pole at $\lambda_{0}$ when $\alpha \geq 1 / 3$. As shown in Fig. 1] for any given $\alpha$, as $w$ decreases from $+\infty$

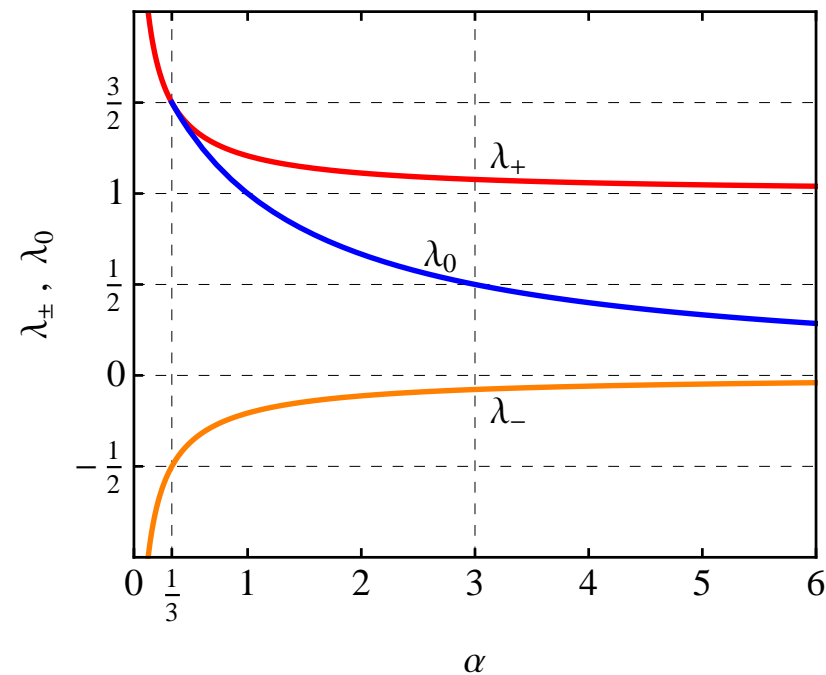

FIG. 2. (Color online). $\lambda_{+}, \lambda_{-}$, and $\lambda_{0}$ are plotted (in red, orange, and blue colors, respectively) against $\alpha$.

to $-\infty$, the saddle point $\lambda^{*}(w)$ moves unidirectionally from $\lambda_{-}$to $\lambda_{+}$on the real $\lambda$ line. For sufficiently large $w$, we have $\lambda_{-}<\lambda^{*}<\lambda_{0}$. In such a situation, the contour of integration can be deformed smoothly through the saddle point $\lambda^{*}$ and therefore we still have $P\left(W_{\tau}=w \tau\right) \sim e^{\tau h_{1}(w)}$. However, as one decreases $w$, at some particular value $w=w^{*}$, the saddle-point hits the singularity - where $w^{*}$ is found by solving $\lambda^{*}\left(w^{*}\right)=$ $\lambda_{0}$ as

$$
w^{*}=\frac{\alpha(\alpha-3)}{3 \alpha-1} .
$$

For $w<w^{*}$, we then have $0<\lambda_{0}<\lambda^{*}$. Therefore, while shifting the contour of integration in the complex- $\lambda$ plane, from its original path along the imaginary- $\lambda$ axis to the steepest descent path through the saddle-point $\lambda^{*}$, a contribution from the pole is picked up according to the residue theorem, which to the leading order is $\exp \left[\tau f_{w}\left(\lambda_{0}\right)\right]$. Now the second derivative of $f_{w}(\lambda)$ along the real- $\lambda$ axis at $\lambda^{*}$ can be found to be

$$
f_{w}^{\prime \prime}\left(\lambda^{*}\right)=\frac{2\left(w^{2}+\alpha\right)^{3 / 2}}{\sqrt{\alpha(1+\alpha)}} .
$$

The above expression is always positive, which means that $f_{w}(\lambda)$ has a minimum at $\lambda^{*}$ along real- $\lambda$. That implies $f_{w}\left(\lambda^{*}\right)<f_{w}\left(\lambda_{0}\right)$. Consequently, the leading saddle-point contribution $\exp \left[\tau f_{w}\left(\lambda^{*}\right)\right]$ is smaller than the leading pole contribution. Therefore, the leading behavior of $P\left(W_{\tau}\right)$ is given by

$$
P\left(W_{\tau}=w \tau\right) \sim e^{\tau h_{2}(w)},
$$

with

$$
h_{2}(w)=f_{w}\left(\lambda_{0}\right) .
$$

By substituting $\lambda_{0}$ in $f_{w}\left(\lambda_{0}\right)$, after some algebra, we find that

$$
h_{2}(w)=\frac{1-\alpha}{1+\alpha}+\frac{2 w}{1+\alpha} .
$$




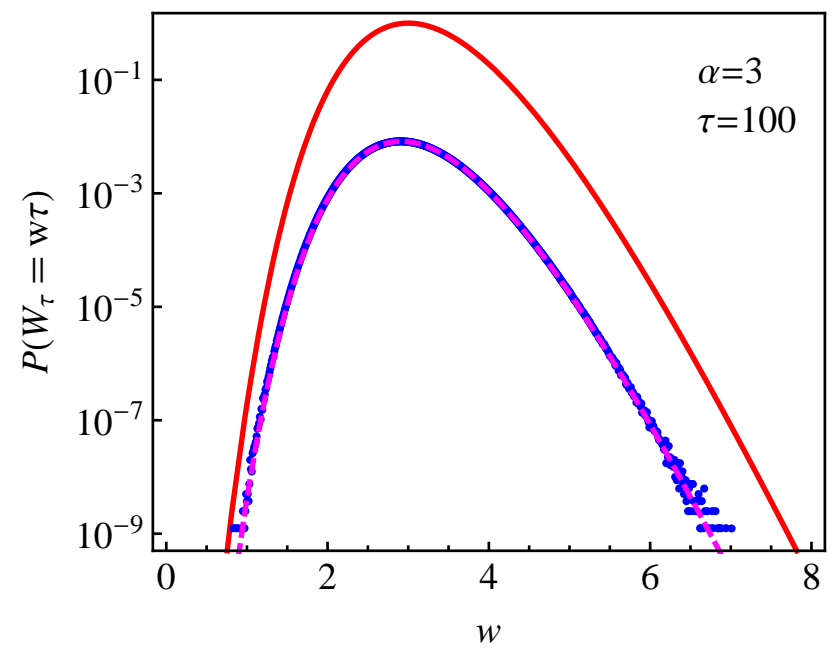

FIG. 3. (Color online). $P\left(W_{\tau}\right)$ against the scaled variable $w=W_{\tau} / \tau$ for $\tau=100$ and $\alpha=3$. The points (blue) are obtained from numerical simulation, and the solid line (red) plots the large deviation form given by Eq. 47). The dashed (magenta) line plots the asymptotic form given by Eq. (51).

To summarize, the large deviation function, defined by

$$
h(w)=\lim _{\tau \rightarrow \infty} \frac{1}{\tau} \ln P\left(W_{\tau}=w \tau\right),
$$

is given by the following. For $\alpha<1 / 3$ :

$$
h(w)=h_{1}(w)
$$

and for $\alpha \geq 1 / 3$ :

$$
h(w)= \begin{cases}h_{1}(w) & \text { for } w \geq w^{*} \\ h_{2}(w) & \text { for } w \leq w^{*}\end{cases}
$$

where $h_{1}(w)$ and $h_{2}(w)$ are given by Eq. (35) and Eq. (43), respectively, and $w^{*}$ is given by Eq. 39. It is easy to show that $h_{1}\left(w^{*}\right)=h_{2}\left(w^{*}\right)$ and $h_{1}^{\prime}\left(w^{*}\right)=h_{2}^{\prime}\left(w^{*}\right)$.

\section{B. Finite time corrections}

In the previous subsection we have obtained the leading behavior of $P\left(W_{\tau}\right)$, which has the large deviation form

$$
P\left(W_{\tau}=w \tau\right) \sim e^{\tau h(w)},
$$

with the large deviation function given by either Eq. (45) or Eq. (46) depending on whether $\alpha<1 / 3$ or $\alpha \geq 1 / 3$. In Fig. 3 we plot this form together with $P\left(W_{\tau}\right)$ that we have obtained from numerical simulation for $\tau=100$. It is evident from the comparison that the large deviation form given by Eq. (47) is not adequate to explain simulation data (or experimental data) of finite time. In this subsection we obtain the sub-leading contributions to the large deviation form.

We have found in the previous subsection that the saddlepoint is located at $\lambda^{*} \in\left(\lambda_{-}, \lambda_{+}\right)$given by Eq. 27). We intend

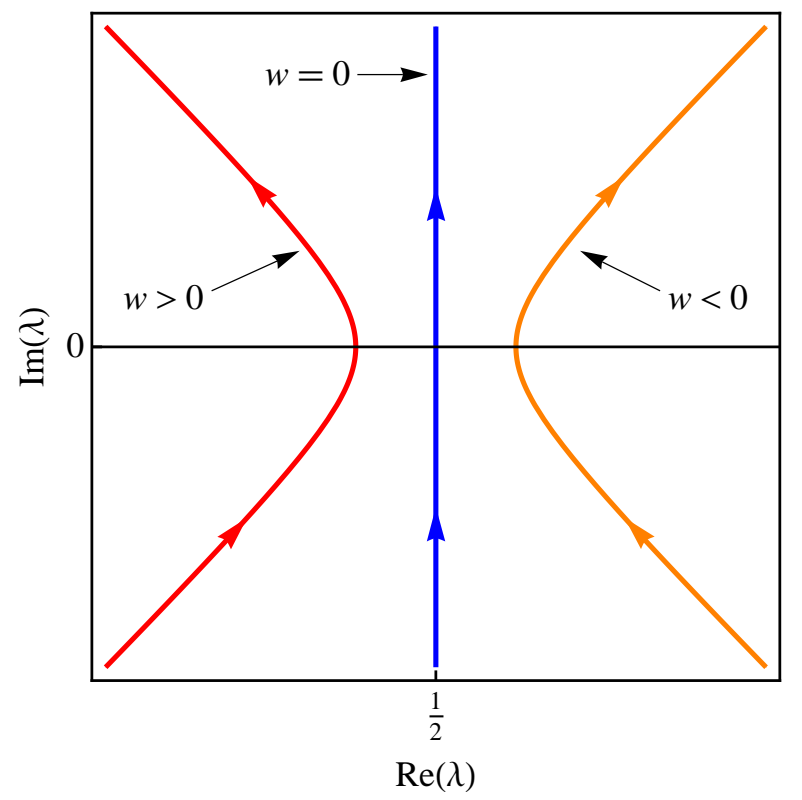

FIG. 4. (Color online). The paths of the steepest descent for $w>0$ (red), $w=0$ (blue) and $w<0$ (orange) respectively.

to deform the original contour of integration in Eq. (25) into the steepest descent path that passes through $\lambda^{*}$ and the imaginary part of $f_{w}(\lambda)$ is constant along the new path. Setting $\operatorname{Im}\left[f_{w}(\lambda)\right]=$ constant $=\operatorname{Im}\left[f_{w}\left(\lambda^{*}\right)\right]=0$ gives the path of the steepest descent as (see Fig. 4 )

$$
\lambda_{R}=\frac{1}{2}\left[1-\frac{w}{\sqrt{\alpha}} \sqrt{\frac{1+\alpha}{w^{2}+\alpha}+4 \lambda_{I}^{2}}\right]
$$

where $\lambda_{R}=\operatorname{Re}(\lambda)$ and $\lambda_{I}=\operatorname{Im}(\lambda)$ are, respectively, the real and imaginary parts of $\lambda$, that is, $\lambda=\lambda_{R}+i \lambda_{I}$. The steepest descent path intersects the real- $\lambda$ axis at $\lambda^{*}$ at an angle $\phi=$ $\pi / 2$, which is evident from Eq. 40, as well as Eq. (48).

\section{Case: $\alpha \leq 1 / 3$}

We have found in Sec. III A that $g(\lambda)$ does not have any singularities in $\left(\lambda_{-}, \lambda_{+}\right)$for $\alpha<1 / 3$. Therefore, we can smoothly deform the contour along the path of the steepest descent through the saddle-point. Subsequently, following the the usual saddle-point approximation method, we write

$$
\int_{-i \infty}^{+i \infty} g(\lambda) e^{\tau f_{w}(\lambda)} d \lambda \approx \frac{\sqrt{2 \pi} g\left(\lambda^{*}\right) e^{\tau f_{w}\left(\lambda^{*}\right)} e^{i \phi}}{\left|\tau f_{w}^{\prime \prime}\left(\lambda^{*}\right)\right|^{1 / 2}}
$$


Using Eqs. 24), 26, 230, 34, 37), 407, and 443, with some algebra, we find that

$$
\begin{aligned}
K(w) & \equiv \frac{\sqrt{2} g\left(\lambda^{*}\right)}{\sqrt{\left|f_{w}^{\prime \prime}\left(\lambda^{*}\right)\right|}} \\
& =\alpha^{3 / 2}(1+1 / \alpha)^{3 / 4}\left(w^{2}+\alpha\right)^{-5 / 4} \\
& \times\left[1+(w+\alpha) \lambda^{*}(w)-h_{1}(w)\right]^{-1} \\
& \times\left[\left\{h_{2}(w)-h_{1}(w)\right\}+(w-\alpha)\left\{\lambda^{*}(w)-\lambda_{0}\right\}\right]^{-1} .
\end{aligned}
$$

It should be noted that, although the last line in the above expression is written in that particular fashion involving $\lambda_{0}$ and $h_{2}(w)$, instead of $\left[1+(w-\alpha) \lambda^{*}(w)-h_{1}(w)\right]^{-1}$, it does not have any singularities for $\alpha<1 / 3$ as we have discussed in the previous subsection. Even for $\alpha=1 / 3$, the above expression diverges only in the limit of $w \rightarrow-\infty$.

Finally, using Eq. (49), and Eq. (50), in (25), we find that (recall that $\phi=\pi / 2$ )

$$
P\left(W_{\tau}=w \tau\right) \approx \frac{K(w)}{2 \sqrt{\pi \tau}} e^{\tau h_{1}(w)} .
$$

It is seen from Fig. 5(a) that the above form is a perfect fit for the data obtained using numerical simulation for $\alpha=1 / 3$.

\section{Case: $\alpha \geq 1 / 3$}

Let us consider the case $\alpha \geq 1 / 3$. We have found in Sec. III A that $g(\lambda)$ for this case has a pole at $\lambda_{0} \in\left(0, \lambda_{+}\right.$] and $\lambda^{*}(w)<\lambda_{0}$ for $w>w^{*}$, whereas $\lambda^{*}(w)>\lambda_{0}$ for $w<w^{*}$. Now if $w \gg w^{*}$, then the asymptotic behavior of Eq. 25] is obtained from the saddle-point approximation, which is given by Eq. 51]. On the other hand, when $w \ll w^{*}$, while deforming the contour of the integration in Eq. (25) to the steepest descent path through the saddle point, it goes around the pole at $\lambda_{0}$ in a clockwise direction. Therefore, according to the residue theorem, the integral pick up a contribution from the pole which is given by $-g_{-1} \exp \left[\tau f_{w}\left(\lambda_{0}\right)\right]$, where

$$
g_{-1}=\lim _{\lambda \rightarrow \lambda_{0}}\left[\left(\lambda-\lambda_{0}\right) g(\lambda)\right]=-\frac{(3 \alpha-1)^{2}}{8 \alpha^{2}(1+\alpha)} .
$$

It is convenient to use Eq. 36 for $g(\lambda)$ to obtain the last expression. The saddle-point contribution to the integral is same as given by Eq. (51). Therefore, combining the contributions from the saddle-point as well as from the pole, we can write that for $\alpha \geq 1 / 3$ when $\left|w-w^{*}\right| \gg 0$,

$$
\begin{aligned}
P\left(W_{\tau}=w \tau\right) & \approx \frac{K(w)}{2 \sqrt{\pi \tau}} e^{\tau h_{1}(w)} \\
& -\theta\left(w^{*}-w\right) g_{-1} e^{\tau h_{2}(w)} .
\end{aligned}
$$

Since $h_{1}\left(w^{*}\right)=h_{2}\left(w^{*}\right)$ and $\lambda^{*}\left(w^{*}\right)=\lambda_{0}$, it is clear from Eq. (50) that $K(w)$ diverges as $w \rightarrow w^{*}$. Therefore, Eq. (53) does not provide the correct description of the actual probability density function as $w$ approaches $w^{*}$ (from any side), which is also seen from Fig. 5. b) and Fig. 5(c).

We carry out an asymptotic analysis of the integral along the path of the steepest descent, which is also valid when $w$ is near $w^{*}$, using the method of uniform asymptotic expansions [40]. The steps are outlined in Appendix B following which we get

$$
P\left(W_{\tau}=w \tau\right) \approx \frac{e^{\tau h_{1}(w)}}{2 \sqrt{\pi \tau}}\left[K(w)-\frac{\operatorname{sgn}\left(w^{*}-w\right) g_{-1}}{\sqrt{h_{2}(w)-h_{1}(w)}}\right]+e^{\tau h_{2}(w)} g_{-1}\left[\frac{\operatorname{sgn}\left(w^{*}-w\right)}{2} \operatorname{erfc}\left(\sqrt{\tau\left[h_{2}(w)-h_{1}(w)\right]}\right)-\theta\left(w^{*}-w\right)\right]
$$

Again, this above asymptotic form matches with simulation results extremely well even for $\tau=10$ as seen from Fig. 5(b) and Fig. 5(c). It follows from Appendix B that the above equation reduces to Eq. (53) for $\left|w-w^{*}\right| \gg 0$. Equation (54) is valid for any values of $w$ including $w=w^{*}$.

\section{REMARKS ON STOCHASTIC INTEGRATION}

Finally, we make some remarks on the evaluation of the integrals of type $\int_{0}^{\tau} f(t) v(t) d t$ - that appears in Eqs. (5) and 20] - in numerical simulations or from experimental data. First the total time interval $(0, \tau)$ is divided into small time intervals of $\Delta t$ duration such that

$$
\int_{0}^{\tau} f(t) v(t) d t=\sum_{n=0}^{N-1} \int_{t}^{t+\Delta t} f\left(t^{\prime}\right) v\left(t^{\prime}\right) d t^{\prime}
$$

where $t=n \Delta t$ and $N \Delta t=\tau$. Next the integral on the right side of the above equation needs to be evaluated for each interval $(t, t+\Delta t)$. At first glance, it might look reasonable to assume that the integrals could be evaluated numerically in simulations or from experimental data by any one of the following approximation schemes:

$$
\begin{aligned}
& \text { (1) } \int_{t}^{t+\Delta t} f\left(t^{\prime}\right) v\left(t^{\prime}\right) d t^{\prime} \approx v(t) F_{\Delta t}(t), \\
& \text { (2) } \int_{t}^{t+\Delta t} f\left(t^{\prime}\right) v\left(t^{\prime}\right) d t^{\prime} \approx \frac{1}{2}[v(t)+v(t+\Delta t)] F_{\Delta t}(t), \\
& \text { (3) } \int_{t}^{t+\Delta t} f\left(t^{\prime}\right) v\left(t^{\prime}\right) d t^{\prime} \approx v(t+\Delta t) F_{\Delta t}(t),
\end{aligned}
$$

where $F_{\Delta t}(t)=\int_{t}^{t+\Delta t} f\left(t^{\prime}\right) d t^{\prime}$, which is of $O(\sqrt{\Delta t})$. However, it turns out that the second one (à la Stratonovich) is the only correct scheme to follow. If one were to use the first scheme instead, the resulting distribution would shift to the left of the true one, whereas the use of the third scheme would result 

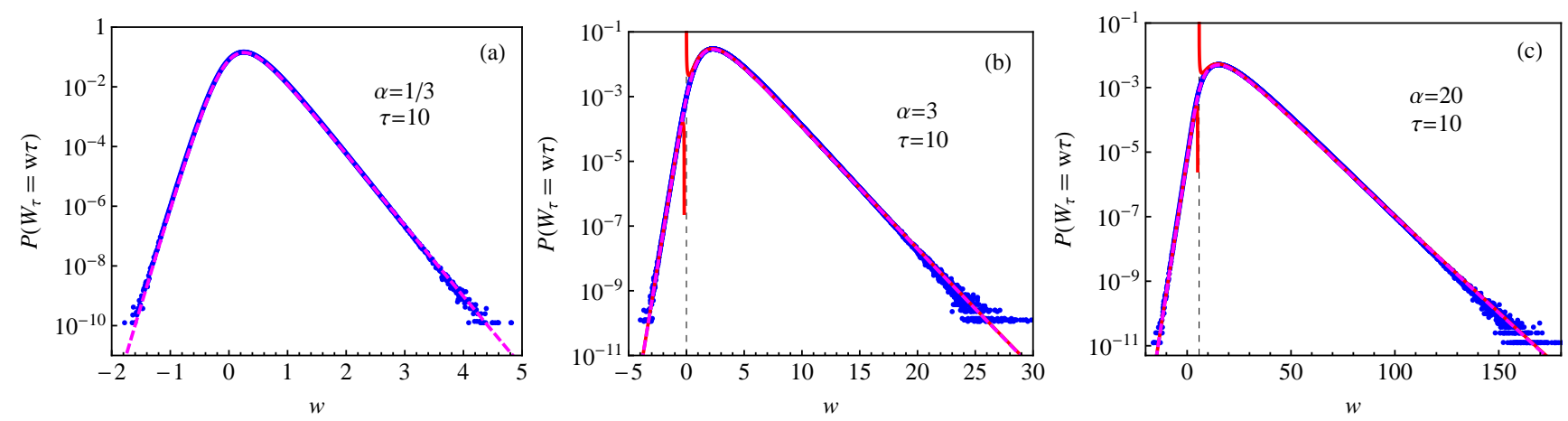

FIG. 5. (Color online). $P\left(W_{\tau}\right)$ against the scaled variable $w=W_{\tau} / \tau$ for $\tau=10$ and (a) $\alpha=1 / 3$, (b) $\alpha=3$, and (c) $\alpha=20$, respectively. The points (blue) are obtained from numerical simulation, and the dashed solid lines (magenta) plot the analytical asymptotic forms given by Eq. (51) for (a) and Eq. (54) for (b) and (c). In (b) and (c): the solid (red) lines plot the form given by Eq. (53), and the dashed vertical lines mark the positions of $w=w^{*}$.

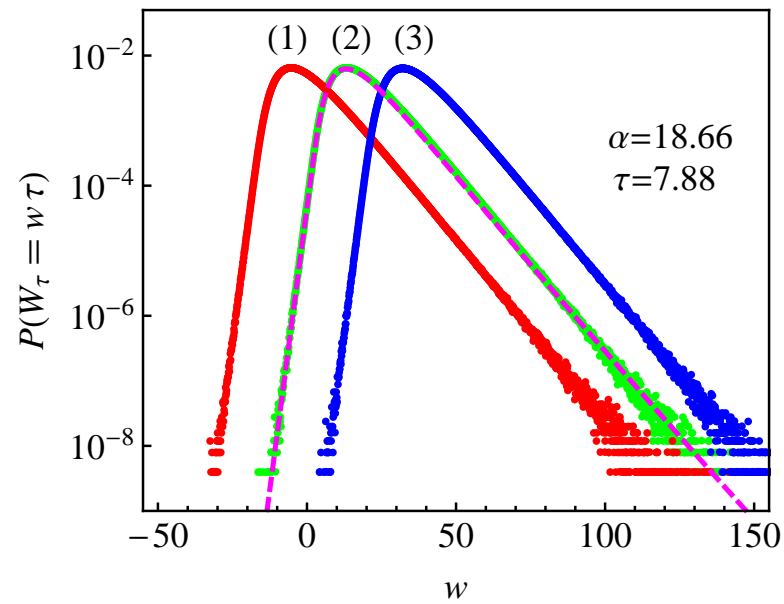

FIG. 6. (Color online) The three sets [(1) red, (2) green and (3) blue] of points plot the probability density functions of the work fluctuations obtained from numerical simulations of the Langavin equation given by Eq. (19), while using the three different numerical integration schemes respectively, outlined in Sec. IV for the work integral given by Eq. 20. The dashed line (magenta) plots the analytical asymptotic forms given by Eq. 54. It is evident that only the second stochastic integration scheme of Sec. IV yields the correct distribution.

in a shift to the right of the actual distribution (see Fig. 6. While comparing our asymptotic form Eq. (54) with the experimental distribution of Ref. [33], the work is evaluated using the second scheme given above [37]. The second scheme is also used while deriving the Fokker-Planck equation given by Eqs. (7) and (8). On the other hand, the methods used here to obtain the solution of the Fokker-Planck equation does not require any such stochastic integration scheme [36]. We have used the Fokker-Planck equation to merely verify the solution. This also proves the correctness of the second scheme in the present context.

\section{SUMMARY}

In this paper, we have applied the recent formalism of Kundu et al. [36] to the case of a harmonically bound Brownian particle coupled to two heat baths at different temperatures. We have considered the fluctuations of the total amount of heat flow $Q$ from one of the baths to the particle in a given time duration $\tau$. Its characteristic function for given initial and final phase-space configurations satisfies a nontrivial Fokker-Planck equation. We have obtained the largest eigenvalue of the Fokker-Planck operator as well as the corresponding eigenfunctions (left and right) exactly. Using those, and integrating over the final configurations and averaging over the initial configurations with respect to the nonequilibrium steady-state, we have obtained the characteristic function $\left\langle e^{-\lambda Q}\right\rangle \approx g(\lambda) \exp [\tau \mu(\lambda)]$ for large $\tau$, where the both $\mu(\lambda)$ and $g(\lambda)$ have been obtained exactly. A special case of this problem corresponds to the work done by an external stochastic force on a harmonic oscillator coupled to a thermal bath. This special case in fact models an recently studied experimental system of a stochastically driven atomic-force microscopy cantilever [33]. For this model we have analyzed the characteristic function and found the exact large deviation function as well as the complete asymptotic forms of the probability density function of the work. We have compared the analytical results with numerical simulations and found excellent agreements between the theory and simulation. In fact, the theoretical asymptotic forms of the probability density function also match quite well with the experimentally obtained forms [37]. Finally, we believe that the results as well as the analytical methods of this paper would be useful for many other similar problems.

\section{ACKNOWLEDGMENTS}

I thank Abhishek Dhar for useful discussions and acknowledge the support of the Indo-French Centre for the Promotion of Advanced Research (IFCPAR/CEFIPRA) under Project 4604-3. 


\section{Appendix A: Fluctuations of the heat flow in a harmonic chain}

Here, for ease of reference, we outline the relevant results, of Ref. [36], for a harmonic chain consisting of $N$ particles connected at its two ends to white-noise Langevin reservoirs at different temperatures $T_{L}$ and $T_{R}$ respectively. The system, described by the variables $X^{T}=\left(x_{1}, x_{2}, \ldots, x_{N}\right)$ and $V^{T}=\left(v_{1}, v_{2}, \ldots, v_{N}\right)$, evolves according to the following equations of motion:

$$
\dot{X}=V, \quad \boldsymbol{M} \dot{V}=-\boldsymbol{\Phi} X-\boldsymbol{\gamma} V+\eta(t),
$$

where $\boldsymbol{M}=\operatorname{diag}\left(m_{1}, m_{2}, \ldots, m_{N}\right)$ is the mass matrix, $\boldsymbol{\Phi}$ is the force matrix, $\boldsymbol{\gamma}$ is the dissipation matrix with elements $\boldsymbol{\gamma}_{i, j}=\delta_{i, j}\left(\delta_{i, 1} \gamma_{L}+\delta_{i, N} \gamma_{R}\right)$, and $\eta$ is the Gaussian noise vector with elements $\eta_{i}(t)=\delta_{i, 1} \eta_{L}(t)+\delta_{i, N} \eta_{R}(t)$ whose correlators are given by Eqs. (2)- (4). The quantity of interest is the total amount of heat flowing from one of the reservoirs - say the left $(\mathrm{L})$ - into the system in a given time duration $\tau$, given by

$$
Q=\int_{0}^{\tau}\left[\eta_{L}(t)-\gamma_{L} v_{1}(t)\right] v_{1}(t) d t
$$

It was found in Ref. [36] that the restricted characteristic function $Z\left(\lambda, U, \tau \mid U_{0}\right)=\left\langle e^{-\lambda Q} \delta[U-U(\tau)]\right\rangle_{U_{0}}$ for fixed initial and final configurations, $U_{0}^{T}=\left(X_{0}^{T}, V_{0}^{T}\right)$ and $U^{T}=$ $\left(X^{T}, V^{T}\right)$, respectively, has the large- $\tau$ asymptotic form

$$
Z\left(\lambda, U, \tau \mid U_{0}\right) \sim \chi\left(U_{0}, \lambda\right) \Psi(U, \lambda) \exp [\tau \mu(\lambda)]
$$

The cumulant generating function $\mu(\lambda)$ is given by

$$
\mu(\lambda)=-\frac{1}{4 \pi} \int_{-\infty}^{\infty} d \omega \ln \left[1+\lambda(\Delta \beta-\lambda) \mathscr{T}_{1}(\omega)\right],
$$

where $\Delta \beta=\left(\gamma_{R} / d_{R}\right)-\left(\gamma_{L} / d_{L}\right)$ and

$$
\mathscr{T}_{1}(\omega)=4 d_{L} d_{R} \omega^{2} \boldsymbol{G}_{1, N}^{+}(\omega) \boldsymbol{G}_{1, N}^{-}(\omega)
$$

with

$$
\boldsymbol{G}^{ \pm}(\omega)=\left[\boldsymbol{\Phi}-\omega^{2} \boldsymbol{M} \pm i \omega \boldsymbol{\gamma}\right]^{-1}
$$

The above formula of $\mu(\lambda)$ has been also generalized to the case of heat conduction across arbitrary harmonic networks [39].

The functions $\Psi(U, \lambda)$ and $\chi\left(U_{0}, \lambda\right)$ have the following Gaussian forms:

$$
\begin{aligned}
\Psi(U, \lambda) & =\frac{\exp \left[-\frac{1}{2} U^{T} \boldsymbol{L}_{1}(\lambda) U\right]}{(2 \pi)^{N} \sqrt{\operatorname{det} \boldsymbol{H}_{1}(\lambda)}} \\
\chi\left(U_{0}, \lambda\right) & =\exp \left[-\frac{1}{2} U_{0}^{T} \boldsymbol{L}_{2}(\lambda) U_{0}\right]
\end{aligned}
$$

where

$$
\begin{aligned}
& \boldsymbol{L}_{1}(\boldsymbol{\lambda})=\boldsymbol{H}_{1}^{-1}+\boldsymbol{H}_{1}^{-1} \boldsymbol{H}_{2}^{T} \\
& \boldsymbol{L}_{2}(\lambda)=-\boldsymbol{H}_{1}^{-1} \boldsymbol{H}_{2}^{T}
\end{aligned}
$$

and $\boldsymbol{H}_{1}$ and $\boldsymbol{H}_{2}$ satisfy the relation

$$
\boldsymbol{H}_{2} \boldsymbol{H}_{1}^{-1} \boldsymbol{H}_{2}^{T}+\boldsymbol{H}_{1}^{-1} \boldsymbol{H}_{2}^{T}=\boldsymbol{H}_{3}
$$

The matrices $\boldsymbol{H}_{1}, \boldsymbol{H}_{2}$, and $\boldsymbol{H}_{3}$ are, respectively, given as follows:

$$
\begin{aligned}
& \boldsymbol{H}_{1}(\lambda)=\frac{1}{2}\left(\boldsymbol{I}_{1}+\boldsymbol{I}_{1}^{T}\right) \quad \text { with } \quad \boldsymbol{I}_{1}(\lambda)=\frac{d_{L} d_{R}}{\pi} \int_{-\infty}^{\infty} d \omega \frac{C_{1,1} F_{2} F_{2}^{\dagger}+C_{1,2} F_{3} F_{2}^{\dagger}+C_{2,1} F_{2} F_{3}^{\dagger}+C_{2,2} F_{3} F_{3}^{\dagger}}{1+\lambda(\Delta \beta-\lambda) \mathscr{T}_{1}(\omega)} \\
& \boldsymbol{H}_{2}(\lambda)=\lim _{\varepsilon \rightarrow 0} \frac{\lambda}{\pi} \int_{-\infty}^{\infty} d \omega e^{i \omega \varepsilon} \frac{d_{L}\left(1-2 i \omega \gamma_{L} \boldsymbol{G}_{1,1}^{+}\right) F_{1}^{*} F_{2}^{\dagger}-2 i \omega\left(\gamma_{L}+\lambda d_{L}\right) d_{R} \boldsymbol{G}_{1, N}^{+} F_{1}^{*} F_{3}^{\dagger}}{1+\lambda(\Delta \beta-\lambda) \mathscr{T}_{1}(\omega)} \\
& \boldsymbol{H}_{3}(\lambda)=\frac{1}{2}\left(\boldsymbol{I}_{3}+\boldsymbol{I}_{3}^{T}\right) \quad \text { with } \quad \boldsymbol{I}_{3}(\lambda)=\frac{\lambda\left(\gamma_{L}+\lambda d_{L}\right)}{\pi} \int_{-\infty}^{\infty} d \omega \frac{F_{1} F_{1}^{\dagger}}{1+\lambda(\Delta \beta-\lambda) \mathscr{T}_{1}(\omega)}
\end{aligned}
$$

In the above expressions $F_{1}, F_{2}$, and $F_{3}$ are column matrices, given respectively by

$$
\begin{aligned}
F_{1}^{T} & =\left(\left[\boldsymbol{G}^{+} \boldsymbol{\Phi}\right]_{1,1},\left[\boldsymbol{G}^{+} \boldsymbol{\Phi}\right]_{1,2}, \ldots,\left[\boldsymbol{G}^{+} \boldsymbol{\Phi}\right]_{1, N},-i \omega\left[\boldsymbol{G}^{+} \boldsymbol{M}\right]_{1,1},-i \omega\left[\boldsymbol{G}^{+} \boldsymbol{M}\right]_{1,2}, \ldots,-i \omega\left[\boldsymbol{G}^{+} \boldsymbol{M}\right]_{1, N}\right), \\
F_{2}^{T} & =\left(G_{1,1}^{+}, G_{2,1}^{+}, \ldots, G_{N, 1}^{+}, i \omega G_{1,1}^{+}, i \omega G_{2,1}^{+}, \ldots, i \omega G_{N, 1}^{+}\right) \\
F_{3}^{T} & =\left(G_{1, N}^{+}, G_{2, N}^{+}, \ldots, G_{N, N}^{+}, i \omega G_{1, N}^{+}, i \omega G_{2, N}^{+}, \ldots, i \omega G_{N, N}^{+}\right)
\end{aligned}
$$

and $C_{i, j}$ is the $\{i, j\}$-th element of the matrix

$$
\boldsymbol{C}(\omega)=\left(\begin{array}{cc}
\frac{1}{d_{R}}-4 \lambda \gamma_{L} \omega^{2}\left|G_{1, N}^{+}\right|^{2} & 4 \lambda \gamma_{L} \omega^{2} G_{1,1}^{+} G_{1, N}^{-}+2 i \lambda \omega G_{1, N}^{-} \\
4 \lambda \gamma_{L} \omega^{2} G_{1,1}^{-} G_{1, N}^{+}-2 i \lambda \omega G_{1, N}^{+} & \frac{1}{d_{L}}+4 \lambda \gamma_{R} \omega^{2}\left|G_{1, N}^{+}\right|^{2}
\end{array}\right) .
$$




\section{The $N=1$ case}

Here we explicitly evaluate the above expressions for the special case of the single Brownian particle in a harmonic potential that is discussed in Sec. II] For this case, we have

$$
G^{ \pm}(\omega)=\left[k-\omega^{2} m \pm i \omega \gamma\right]^{-1}
$$

which gives

$$
\mathscr{T}_{1}(\omega)=\frac{4 d_{L} d_{R} \omega^{2}}{\left(k-m \omega^{2}\right)^{2}+\gamma^{2} \omega^{2}} .
$$

In this case Eq. (A4) can be evaluated explicitly and the final expression is given by Eq. 10.

To obtain the explicit forms of of the functions given by Eqs. A7] and A8, we first find that

$$
F_{1}=G^{+}\left(\begin{array}{c}
k \\
-i \omega m
\end{array}\right) \text { and } F_{2}=F_{3}=G^{+}\left(\begin{array}{c}
1 \\
i \omega
\end{array}\right)
$$

Moreover, adding the elements of $\boldsymbol{C}(\omega)$ from Eq. A18 and then using the identity $-i\left[G^{-}-G^{+}\right]=2 \gamma \omega\left|G^{+}\right|^{2}$ we get $C_{1,1}+C_{1,2}+C_{2,1}+C_{2,2}=d_{L}^{-1}+d_{R}^{-1}$.

Next, we carry out the integrations in Eqs. (A12)-(A14) to find that

$$
\begin{aligned}
\boldsymbol{H}_{1}(\lambda) & =\frac{d_{L}+d_{R}}{\gamma \eta(\lambda)}\left(\begin{array}{cc}
1 / k & 0 \\
0 & 1 / m
\end{array}\right), \\
\boldsymbol{H}_{2}(\lambda) & =\frac{\lambda d_{L}-(\gamma / 2)[\eta(\lambda)-1]}{\gamma \eta(\lambda)}\left(\begin{array}{ll}
1 & 0 \\
0 & 1
\end{array}\right), \\
\boldsymbol{H}_{3}(\lambda) & =\frac{\lambda\left(\gamma_{L}+\lambda d_{L}\right)}{\gamma \eta(\lambda)}\left(\begin{array}{cc}
k & 0 \\
0 & m
\end{array}\right) .
\end{aligned}
$$

It can be checked that the above matrices satisfy the relation given by Eq. A11.

Using the above matrices in Eqs. (A9) and (A10, respectively, we obtain

$$
\begin{aligned}
& \boldsymbol{L}_{1}(\boldsymbol{\lambda})=B_{+}(\lambda)\left(\begin{array}{cc}
k & 0 \\
0 & m
\end{array}\right), \\
& \boldsymbol{L}_{2}(\boldsymbol{\lambda})=B_{-}(\boldsymbol{\lambda})\left(\begin{array}{cc}
k & 0 \\
0 & m
\end{array}\right),
\end{aligned}
$$

where

$$
B_{ \pm}(\lambda)=\frac{\gamma \eta(\lambda) \pm\left(\gamma+2 \lambda d_{L}\right)}{2\left(d_{L}+d_{R}\right)}
$$

Finally, using the above results in Eq. A7] and Eq. A8, we obtain Eq. (11) and Eq. (12) respectively.

\section{Appendix B: Uniform asymptotic expansions for a saddle-point near a pole}

Following Ref. [40], we outline below the method of uniform asymptotic expansion of a integral having a saddle-point near a pole. Let us denote

$$
I(\tau)=\int_{C} g(\lambda) e^{\tau f_{w}(\lambda)} d \lambda,
$$

where $C$ denotes the contour along the path of steepest descent. We set

$$
f_{w}(\lambda)-f_{w}\left(\lambda^{*}\right)=-u^{2} .
$$

Since the imaginary part of $f_{w}(\lambda)$ is constant (which happens to be zero in our particular case) on $C$, the above integral can be converted into an integral with respect to a real variable $u$, as

$$
I(\tau)=e^{\tau f_{w}\left(\lambda^{*}\right)} \int_{-\infty}^{\infty} q(u) e^{-\tau u^{2}} d u
$$

with

$$
q(u)=g(\lambda) \frac{d \lambda}{d u} .
$$

The pole $\lambda_{0}$ of $g(\lambda)$ is then mapped to a pole of $q(u)$ at $u=i b$ with

$$
b=\operatorname{sgn}\left(\lambda^{*}-\lambda_{0}\right) \sqrt{f_{w}\left(\lambda_{0}\right)-f_{w}\left(\lambda^{*}\right)} .
$$

Note that, $f_{w}(\lambda)$ is minimum at $\lambda^{*}$ along real- $\lambda$. Therefore, $f_{w}\left(\lambda_{0}\right)>f_{w}\left(\lambda^{*}\right)$, and hence $b$ is real. We can write

$$
q(u)=\frac{c_{-1}}{u-i b}+\psi(u)
$$

where $c_{-1}=\lim _{u \rightarrow i b}[(u-i b) q(u)]$, and $\psi(u)$ has no pole at $u=i b$ or $u=0$. To evaluate $c_{-1}$ we note that $u^{2}+$ $b^{2}=f_{w}\left(\lambda_{0}\right)-f_{w}(\lambda)$ and $d \lambda / d u=-2 u / f^{\prime}(\lambda)$. Moreover, $f_{w}(\lambda)=f_{w}\left(\lambda_{0}\right)+\left(\lambda-\lambda_{0}\right) f^{\prime}\left(\lambda_{0}\right)+\cdots$ as $\lambda \rightarrow \lambda_{0}$. Therefore, we get

$$
\begin{aligned}
c_{-1} & =\lim _{u \rightarrow i b}\left[\left(\frac{u^{2}+b^{2}}{u+i b}\right)\left(\frac{d \lambda}{d u}\right) g(\lambda)\right] \\
& =\lim _{\lambda \rightarrow \lambda_{0}}\left[\left(\lambda-\lambda_{0}\right) g(\lambda)\right]=g_{-1},
\end{aligned}
$$

where $g_{-1}$ is given by Eq. (52).

Let us first look at the integral

$$
J_{1}(\tau)=\int_{-\infty}^{\infty} \frac{e^{-\tau u^{2}}}{u-i b} d u
$$

It satisfies the differential equation

$$
J_{1}^{\prime}(\tau)-b^{2} J_{1}(\tau)=-i b \sqrt{(\pi / \tau)},
$$

with the boundary condition $J_{1}(\infty)=0$. It can be verified that the solution is given by

$$
J_{1}(\tau)=\operatorname{sgn}(b) i \pi e^{\tau b^{2}} \operatorname{erfc}(\sqrt{\tau}|b|) .
$$

Next, we look at the integral

$$
J_{2}(\tau)=\int_{-\infty}^{\infty} \psi(u) e^{-\tau u^{2}} d u .
$$


Expanding $\psi(u)$ Taylor series about $u=0 \quad \psi(u)=$ $\sum_{n=0}^{\infty} \psi^{(n)}(0) u^{n} / n$ ! in the above integral, and then term by term integration of the series yields

$$
J_{2}(\tau)=\sum_{m=0}^{\infty} \frac{\psi^{(2 m)}(0)}{(2 m) !} \frac{\Gamma(m+1 / 2)}{\tau^{m+1 / 2}} .
$$

In terms of $J_{1,2}(\tau)$ we finally have

$$
I(\tau)=e^{\tau f_{w}\left(\lambda^{*}\right)}\left[g_{-1} J_{1}(\tau)+J_{2}(\tau)\right]
$$

In the following, we consider the large $\tau$ limit and find the leading order contribution of $J_{2}(\tau)$, i.e.,

$$
J_{2}(\tau)=\psi(0) \sqrt{(\pi / \tau)}+O\left(\tau^{-3 / 2}\right)
$$

From Eq. B6 we get

$$
\psi(0)=q(0)+\frac{g-1}{i b}
$$

and then from Eq. (B4)

$$
q(0)=\left.g\left(\lambda^{*}\right) \frac{d \lambda}{d u}\right|_{\lambda \rightarrow \lambda^{*}}
$$

Near $\lambda^{*}$ we have

$$
-u^{2}=\frac{1}{2} f^{\prime \prime}\left(\lambda^{*}\right)\left(\lambda-\lambda^{*}\right)^{2}+\cdots .
$$

Let $\lambda-\lambda^{*}=r e^{i \phi}$ and $f^{\prime \prime}\left(\lambda^{*}\right)=\left|f^{\prime \prime}\left(\lambda^{*}\right)\right| e^{i \theta}$. Therefore,

$$
-u^{2}=\frac{r^{2}}{2}\left|f^{\prime \prime}\left(\lambda^{*}\right)\right| e^{i(\theta+2 \phi)}+\cdots
$$

Since, $u$ is real, we have $\theta+2 \phi=\pi$, and

$$
u= \pm \frac{r}{\sqrt{2}}\left|f^{\prime \prime}\left(\lambda^{*}\right)\right|^{1 / 2}+\cdots
$$

Thus,

$$
\left.\frac{d u}{d \lambda}\right|_{\lambda \rightarrow \lambda^{*}}=\left.\frac{d u}{d r} \frac{d r}{d \lambda}\right|_{\lambda \rightarrow \lambda^{*}}=\frac{1}{\sqrt{2}}\left|f^{\prime \prime}\left(\lambda^{*}\right)\right|^{1 / 2} e^{-i \phi} .
$$

Substitution of this into Eq. B16 yields

$$
q(0)=\frac{\sqrt{2} g\left(\lambda^{*}\right)}{\sqrt{\left|f_{w}^{\prime \prime}\left(\lambda^{*}\right)\right|}} e^{i \phi} .
$$

In our case, from Eq. (40) we get $\theta=0$, and therefore $\phi=$ $\pi / 2$.

If the saddle-point and the pole are far apart, then using $J_{1}(\tau) \sim(i / b) \sqrt{(\pi / \tau)}$ for large $b$ and $\tau$, it is immediately checked that $I(\tau)$ reduces to the usual saddle-point approximation given by Eq. (49). More generally, $I(\tau)$ given by Eq. B13, with $J_{1,2}(\tau)$ given by Eqs. (B10) and (B12) respectively, is valid for all $b$, including the limit $b \rightarrow 0$. For large $\tau$, using Eqs. (B14), (B15) and (B21) we get

$$
J_{2}(\tau)=i \sqrt{\frac{\pi}{\tau}}\left[\frac{\sqrt{2} g\left(\lambda^{*}\right)}{\sqrt{\left|f_{w}^{\prime \prime}\left(\lambda^{*}\right)\right|}}-\frac{g_{-1}}{b}\right]+O\left(\tau^{-3 / 2}\right) .
$$

[1] D. J. Evans, E. G. D. Cohen, and G. P. Morriss, Phys. Rev. Lett. 71, 2401 (1993); D. J. Evans and D. J. Searles, Phys. Rev. E 50, 1645 (1994).

[2] G. Gallavotti and E. G. D. Cohen, Phys. Rev. Lett. 74, 2694 (1995); J. Stat. Phys. 80, 931 (1995).

[3] C. Jarzynski, Phys. Rev. Lett. 78, 2690 (1997).

[4] J. Kurchan, J. Phys. A: Math. Gen. 31, 3719 (1998).

[5] J. L. Lebowitz and H. Spohn, J. Stat. Phys. 95, 333 (1999).

[6] G. E. Crooks, Phys. Rev. E 60, 2721 (1999).

[7] T. Hatano and S. Sasa, Phys. Rev. Lett. 86, 3463 (2001).

[8] J. Farago, J. Stat. Phys., 107, 781 (2002).

[9] R. van Zon and E. G. D. Cohen, Phys. Rev. Lett. 91, 110601 (2003); Phys. Rev. E 67, 046102 (2003); Phys. Rev. E 69, 056121 (2004).

[10] R. van Zon, S. Ciliberto, and E. G. D. Cohen, Phys. Rev. Lett. 92, 130601 (2004).

[11] O. Mazonka and C. Jarzynski, e-print arXiv:condmat/9912121

[12] O. Narayan and A. Dhar, J. Phys. A 37, 63 (2004).

[13] T. Bodineau and B. Derrida, Phys. Rev. Lett. 92, 180601 (2004).

[14] U. Seifert, Phys. Rev. Lett. 95, 040602 (2005).

[15] M. Baiesi, T. Jacobs, C. Maes, and N. S. Skantzos, Phys. Rev. E 74, 021111 (2006);

[16] F. Bonetto, G. Gallavotti, A. Giuliani, and F. Zamponi, J. Stat. Phys 123, 39 (2006).
[17] P. Visco, J. Stat. Mech. (2006) P06006.

[18] K. Saito and A. Dhar Phys. Rev. Lett. 99, 180601 (2007).

[19] R. J. Harris and G. M. Schütz, J. Stat. Mech. (2007) P07020;

[20] G. M. Wang, E. M. Sevick, E. Mittag, D. J. Searles, and D. J. Evans, Phys. Rev. Lett. 89, 050601 (2002).

[21] G. M. Wang, J. C. Reid, D. M. Carberry, D. R. M. Williams, E. M. Sevick, and D. J. Evans, Phys. Rev. E 71, 046142 (2005).

[22] D. M. Carberry, J. C. Reid, G. M. Wang, E. M. Sevick, D. J. Searles, and D. J. Evans, Phys. Rev. Lett. 92, 140601 (2004).

[23] W. I. Goldburg, Y. Y. Goldschmidt, and H. Kellay, Phys. Rev. Lett. 87, 245502 (2001).

[24] K. Feitosa and N. Menon, Phys. Rev. Lett. 92, 164301 (2004).

[25] N. Garnier and S. Ciliberto, Phys. Rev. E 71, 060101 (2005).

[26] J. Liphardt et al., Science 296, 1832 (2002).

[27] D. Collin et al., Nature 437, 231 (2005).

[28] S. Majumdar and A. K. Sood, Phys. Rev. Lett. 101, 078301 (2008).

[29] F Douarche, S. Joubaud, N. B. Garnier, A. Petrosyan, and S. Ciliberto, Phys. Rev. Lett. 97, 140603 (2006).

[30] E. Falcon, S. Aumaitre, C. Falcon, C. Laroche, and S. Fauve, Phys. Rev. Lett. 100, 064503 (2008).

[31] M. Bonaldi et al., Phys. Rev. Lett. 103, 010601 (2009).

[32] S Ciliberto, S Joubaud and A Petrosyan, J. Stat. Mech. (2010) P12003.

[33] J. R. Gomez-Solano, L. Bellon, A. Petrosyan and S. Ciliberto, 
EPL 89, 60003 (2010).

[34] H. Touchette, Phys. Rep. 478, 1 (2009).

[35] B. Derrida and E. Brunet, Einstein Aujourdhui (Les Ulis: EDP Sciences, 2005).

[36] A. Kundu, S. Sabhapandit and A. Dhar, J. Stat. Mech. (2011) P03007.
[37] S. Sabhapandit, EPL 96, 20005 (2011).

[38] H. C. Fogedby and A. Imparato, J. Stat. Mech. (2011) P05015.

[39] K. Saito and A. Dhar, Phys. Rev. E 83, 041121 (2011).

[40] R. Wong, Asymptotic Approximations of Integrals, (SIAM, Philadelphia, PA, 2001). 